\title{
Vorwort BHM
}

\author{
Helmut Antrekowitsch \\ Lehrstuhl für Nichteisenmetallurgie, Montanuniversität Leoben, Österreich
}

Online publiziert am 3. April 2013

Die Problematik der Verfügbarkeit von Rohstoffen ist mittlerweile auch in Europa das beherrschende Thema unterschiedlicher Branchen und politischer Entscheidungsträger. Unter immer schwierigeren Rahmenbedingungen sieht sich die produzierende Industrie mit Herausforderungen konfrontiert, die ein ganzheitliches Vorgehen bei vielfach konkurrierenden Zielvorgaben erforderlich machen. Gerade Nichteisenmetalle, wie Aluminium, Kupfer, Zink, Zinn, Edel- und Refraktärmetalle sowie die Seltenen Erden, stehen bei der Produktion von Hightech-Produkten im Mittelpunkt der Betrachtung. Da im Vergleich zu anderen Kontinenten Europa allerdings nur begrenzte Primärrohstoffe besitzt, kommt dem Recycling eine immer größere Bedeutung zu. Der enorme Anfall von Sekundärmaterialien sowie die Rohstoffabhängigkeit führen unweigerlich zu der Notwendigkeit, diese sekundären Ressourcen wiederum der Wertschöpfungskette im Rahmen einer optimierten Kreislaufwirtschaft zuzuführen und nicht zu exportieren oder zu deponieren. Sowohl primär- als auch sekundärmetallurgisch sind daher innovative und verbesserte Verfahren zu entwickeln, um den rohstofflichen Engpässen entgegenzuwirken. Da jedoch ein überdurchschnittlich hoher Anteil an werthaltigen Sekundärmaterialien am Ende ihres Produkt- lebenszyklus den europäischen Kreislauf verlässt, sind auch gesetzliche Rahmenbedingungen seitens der Politik unerlässlich. Die Montanuniversität stellt sich mit ihrer Positionierung entlang der Wertschöpfungskette seit Jahrzehnten erfolgreich diesen Herausforderungen und konnte so, in Kooperation mit Industrie- sowie Forschungspartnern österreichweit eine führende Position einnehmen.

In diesem Zusammenhang spielt die Vernetzung der Nichteisenmetallurgie mit der Werkstofftechnik eine entscheidende Rolle. Die Entwicklung neuer Materialien führt unweigerlich, neben den geforderten Eigenschaften, auch zur Frage von Nachhaltigkeit, Energieeinsparung sowie Recycling. In dieser vorliegenden Ausgabe werden im Besonderen die erwähnten Themenstellungen angesprochen und zeigen die Vielfältigkeit der Forschungsgebiete im Bereich der Nichteisenmetalle. Gerade das Recycling und die damit verbundene Materialentwicklung stellen eine Zukunftsdisziplin dar, weshalb sich auch die Montanuniversität Leoben in vielen unterschiedlichen Schwerpunkten mit dieserThematik beschäftigt.

Helmut Antrekowitsch

Gastherausgeber
Johannes Schenk Herausgeber

Prof. H. Antrekowitsch ( $\square$ ) 